\title{
Reductive evolution and unique infection and feeding mode in the CPR predatory bacterium Vampirococcus lugosii
}

David Moreira $^{1}$, Yvan Zivanovic ${ }^{2}$, Ana I. López-Archilla ${ }^{3}$, Miguel Iniesto ${ }^{1}$ and Purificación $5 \quad$ López-García ${ }^{1}$

${ }^{1}$ Ecologie Systématique Evolution, CNRS, Université Paris-Saclay, AgroParisTech, Orsay, France. ${ }^{2}$ Institut de Biologie Intégrative de la Cellule, CNRS, Université Paris-Saclay, Orsay, France. ${ }^{3}$ Departamento de Ecología, Universidad Autónoma de Madrid, Madrid, Spain.

10 e-mail: david.moreira@u-psud.fr

Keywords: CPR bacteria, Patescibacteria, predatory bacteria, genome reduction, CRISPR-Cas. 
15 The Candidate Phyla Radiation (CPR) constitutes a large supergroup of mostly uncultured bacterial lineages discovered through metabarcoding and metagenomics in diverse environments. Having small cell sizes, reduced genomes, and limited biosynthetic capabilities, they are thought to be symbionts of other organisms from which they obtain essential biomolecules. However, the nature of this symbiosis (mutualistic, neutral, or

20 parasitic) has been ascertained only for rare cultured members of the CPR phylum Saccharibacteria, which are epibiotic parasites of other bacteria. Here, we characterize the biology and the genome of Vampirococcus lugosii, which becomes the first described species of Vampirococcus, a genus of epibiotic bacteria morphologically identified decades ago. Vampirococcus belongs to the CPR phylum Absconditabacteria. It feeds on anoxygenic

25 photosynthetic gammaproteobacteria, fully absorbing their cytoplasmic content. The cells divide epibiotically, forming multicellular stalks whose apical cells can more easily reach new hosts. Vampirococcus genome is small (1.3 Mbp) and highly reduced in biosynthetic metabolism genes. However, it is enriched in genes related to an elaborate, fibrous cell surface likely involved in complex interactions with the host. Comparative genomic

30 analyses show that gene loss has been continuous during Absconditabacteria, and generally most CPR bacteria, evolution. Nonetheless, gene loss was compensated by gene acquisition by horizontal gene transfer and evolution de novo. In Vampirococcus, these innovations include new CRISPR-Cas effectors and a novel electron transport chain. Our findings confirm parasitism as a widespread lifestyle of CPR bacteria, which probably play a 35 previously neglected virus-like ecological role in ecosystems, controlling bacterial populations by a unique form of predation. 


\section{Introduction}

40 Most prokaryotic organisms have remained unknown to science for a long time because of their limited morphological diversity and our inability to culture them. However, over the past few decades, the increasing use of molecular methods opened the black box of prokaryotic diversity ${ }^{1}$. Metabarcoding based on 16S rRNA gene PCR-amplification and sequencing (via, initially, Sanger and, more recently, diverse high-throughput sequencing techniques), unveiled a huge

45 diversity of prokaryotes across ecosystems, including many high-rank (e.g. phylum or class level) clades for which we still lack cultured representatives. Direct, PCR-independent, massive sequencing of environmental DNA (metagenomics) completed this picture by i) revealing lineages whose rRNA genes escaped PCR amplification and ii) providing data about the complete gene complement of microbial communities and, hence, about their metabolic

50 potential ${ }^{1-4}$. The possibility to assemble genome sequences from single cell amplified genomes ${ }^{5}$ or by binning from complex metagenomes ${ }^{6}$ has further led to gain genome-based knowledge for specific uncultured groups. Some of these groups are widely diverse and/or have pivotal importance in evolution, such as the eukaryote-related Asgard archaea ${ }^{7}$. Another such group is the 'Candidate Phyla Radiation' (CPR), which encompasses several dozens of high-rank lineages

55 that represent a substantial diversity of the bacterial domain ${ }^{2,8}$.

Genome-resolved metagenomics has allowed the reconstruction of complete genome sequences for many CPR lineages. Despite the impressive diversity of this group, a clear common pattern distills from these sequences: all CPR bacteria contain small genomes (often $<1$ $\mathrm{Mb}$ ) that encode limited metabolic capacities ${ }^{3,8,9}$. Most CPR bacteria are inferred to be obligatory

60 anaerobes that ferment different substrates, lacking aerobic or anaerobic respiration. In most cases, their small genomes also lack genes involved in basic biosynthetic capacities, notably of lipids, nucleotides, and most amino acids ${ }^{3,8}$. These characteristics mirror those found in the DPANN archaea, which also exhibit small genomes and simplified metabolisms ${ }^{9,10}$. Consistent with their reduced genomes, CPR bacteria consist of very small cells when observed under the 65 microscope, and are often able to pass through $0.2 \mu \mathrm{m}$ pore-diameter filters ${ }^{11}$. The cultivation of the first species belonging to the CPR clade (specifically to the phylum Saccharibacteria, previously known as TM7) showed its cells growing attached to the surface of the humanassociated actinobacterium Actinomyces odontolyticus ${ }^{12}$. More recently, three additional 
Saccharibacteria species growing in epibiotic association with diverse Actinobacteria of the

70 human oral microbiome have been isolated ${ }^{13}$. However, although there is increasing support for the ultra-small CPR bacteria being epibionts that depend on hosts with more complete biosynthetic repertoires, current evidence remains fragmentary and based on these very few documented species of Saccharibacteria. Important questions such as the generality and nature (beneficial, neutral or deleterious) of CPR bacteria-host interactions need to be addressed on

75 additional species to get global insight on the biology of this bacterial supergroup.

Here, we present the in-depth characterization of Candidatus Vampirococcus lugosii, a new CPR species from the phylum Absconditabacteria (previously known as SR1) found in the athalassic salt lake Salada de Chiprana. The genus Vampirococcus was described several decades ago as one of the rare examples of predatory bacteria ${ }^{14}$, but its phylogenetic identity remained

80 elusive until now. Our observation of living plankton from this lake showed that Ca. V. lugosii grows and characteristically divides multiple times attached to cells of its specific host, the anoxygenic photosynthetic gammaproteobacterium Halochromatium sp., until it completely absorbs its cytoplasmic content thereby killing it. Ca. V. lugosii has a small genome (1.3 Mb), coding for an extremely limited biosynthetic metabolism but for an elaborate cell surface, most

85 likely involved in a complex interaction with the host. These findings suggest that parasitism is widespread in CPR bacteria and that they play a previously neglected virus-like ecological role in controlling bacterial populations in many ecosystems.

\section{Results and Discussion}

90 Identification of ultra-small cells associated with blooms of anoxygenic phototrophic gammaproteobacteria

The Salada de Chiprana (NE Spain) is the only permanent athalassic hypersaline lake in Western Europe. It harbors thick, conspicuous microbial mats covering its bottom (Fig. 1a-b) and exhibits periodic stratification during which the deepest part of the water column becomes anoxic and

95 sulfide-rich, favoring the massive development of sulfide-dependent anoxygenic photosynthetic bacteria $^{15}$. We collected microbial mat fragments that were maintained in culture in the laboratory. After several weeks, we observed a bloom of anoxygenic photosynthetic bacteria containing numerous intracellular sulfur granules (Fig. 1c). Many of these bacterial cells showed 
one or several much smaller and darker cells attached to their surface (Fig. 1d-e). These cells

100 were highly mobile, swimming at high speed with frequent changes of direction (Supplementary Video 1), in contrast with the non-infected cells that displayed slower (approximately half speed) and more straight swimming. Sometimes, two or more photosynthetic cells were connected through relatively short filaments formed by stacked epibiont cells (Fig. 1f). Although the photosynthetic cells carrying these epibionts were often alive and actively swimming, in some 105 cases epibionts were associated to empty ghost cells where only the photosynthesis-derived sulfur granules persisted. Closer scrutiny of the epibionts revealed that they actually consisted of piles of up to 10 very flattened cells of 500-600 nm diameter and 200-250 nm height (e.g., Fig. 1d). These characteristics (size, morphology, and specific attachment to sulfide-dependent anoxygenic photosynthetic bacteria) perfectly matched the morphological description of the 110 genus Vampirococcus observed over forty years ago in sulfidic freshwater lakes ${ }^{14}$.

Since the first Vampirococcus description included transmission electron microscopy (TEM) images, to further ascertain this identification we examined our Chiprana Lake samples under TEM and scanning electron microscopy (SEM). SEM images confirmed the peculiar structure of the epibionts, with multiple contiguous cells separated by deep grooves (Fig. 1g). Thin sections

115 observed under TEM confirmed that the cells were actually separated by a space of $\sim 20-50 \mathrm{~nm}$ filled by a fibrous material (Fig. 1h-i). The space between epibiont and host cells was larger $(\sim 100 \mathrm{~nm})$ and also filled by dense fibrous material (Fig. 1h). The sections also showed that, in contrast with the typical Gram-negative double membrane structure of the host, the epibiont cells had a single membrane surrounded by a thick layer of fibrous material that conferred a rugose 120 aspect to the cells (Fig. 1i). In sharp contrast with the often highly vacuolated cytoplasm of the host, the epibiont cells showed a dense, homogeneous content. These observations were also in agreement with those published for Vampirococcus, reinforcing our conclusion that the epibionts we observed belonged to this genus, although most likely to a different species, as the first Vampirococcus described occurred in a non-hypersaline lake ${ }^{14}$.

125 Using a micromanipulator coupled to an inverted microscope, we collected cells of the anoxygenic photosynthetic bacterium carrying Vampirococcus attached to their surface (Extended Data Fig. 1) and proceeded to amplify, clone, and sequence their 16S rRNA genes. We were able to obtain sequences for both the epibiont and the host for ten infected cells and, in all cases, we retrieved the same two sequences. The host was found to be a Halochromatium-like 
130 gammaproteobacterium (Extended Data Fig. 2). Phylogenetic analysis of the epibiont sequence showed that it branched within the CPR radiation close to the Absconditabacteria (Extended Data Fig. 3), previously known as candidate phylum SR $1^{2}$. Since all host and epibiont cells we analyzed had identical 16S rRNA gene sequences, suggesting that they were the result of a clonal bloom, we collected three sets of ca. 10 infected cells and carried out whole genome 135 amplification (WGA) before sequencing (Illumina HiSeq; see Methods). This strategy allowed us to assemble the nearly complete genome sequence of the Vampirococcus epibiont (see below). In contrast with the completeness of this genome, we only obtained a very partial assembly $(\sim 15 \%)$ of the host genome, probably because of the consumption of the host DNA by the epibiont. To make more robust phylogenetic analyses of Vampirococcus, we retrieved the protein

140 sequence set used by Hug et al. to reconstruct a multi-marker large-scale phylogeny of bacteria ${ }^{2}$. The new multi-gene maximum likelihood (ML) phylogenetic tree confirmed the affiliation of our Vampirococcus species to the Absconditabacteria with maximum support, and further placed this clade within a larger well-supported group also containing the candidate phyla Gracilibacteria and Peregrinibacteria (Fig. 2a and Extended Data Fig. 4). Therefore, our epibiotic bacterium

145 represents the first characterized member of this large CPR clade and provides a phylogenetic identity for the predatory bacterial genus Vampirococcus described several decades ago. We propose to call this new species Candidatus Vampirococcus lugosii (see Taxonomic Appendix).

\section{Genomic evidence of adaptation to predatory lifestyle}

150 We sequenced DNA from three WGA experiments corresponding each to 10 HalochromatiumVampirococcus consortia. Many of the resulting (57.2 Mb) raw sequences exhibited similarity to those of available Absconditabacteria/SR1 metagenome-assembled genomes (MAGs) and, as expected, some also to Gammaproteobacteria (host-derived sequences) as well as a small proportion of potential contaminants probably present in the original sample (Bacillus- and

155 fungi-like sequences). To bin the Vampirococcus sequences out of this mini-metagenome, we applied tetranucleotide frequency analysis on the whole sequence dataset using emergent selforganizing maps $(\mathrm{ESOM})^{6}$. One of the ESOM sequence bins was enriched in Absconditabacteria/SR1-like sequences and corresponded to the Vampirococcus sequences, which we extracted and assembled independently. This approach yielded an assembly of 
$1601,310,663 \mathrm{bp}$. We evaluated its completeness by searching i) a dataset of 40 universally distributed single-copy genes ${ }^{16}$ and ii) a dataset of 43 single-copy genes widespread in CPR bacteria $^{8}$. We found all them as single-copy genes in the Vampirococcus genome, with the exception of two signal recognition particle subunits from the first dataset which are absent in many other CPR bacteria ${ }^{17}$. These results supported that the Vampirococcus genome assembly 165 was complete and had no redundancy. Manually curated annotation predicted 1,151 proteincoding genes, a single rRNA gene operon, and 38 tRNA coding genes. As already found in other Absconditabacteria/SR1 genomes ${ }^{18}$, the genetic code of Vampirococcus is modified, with the stop codon UGA reassigned as an additional glycine codon.

A very large proportion of the predicted proteins (48.95\%) had no similarity to any COG 170 class and lacked any conserved domain allowing their functional annotation (Fig. 2b). Thus, as for other CPR bacteria, a significant part of their cellular functions remains inaccessible. A comparison with three other Absconditabacteria genomes revealed a very small set of only 390 genes conserved in all them (Fig. 2c), suggesting a highly dynamic evolution of gene content in these species. Comparison with more distantly related CPR groups (Gracilibacteria and

175 Peregrinibacteria) showed that gene loss has been a dominant trend in all these organisms, which have lost $30-50 \%$ of the $\sim 1100$ genes inferred to have existed in their last common ancestor (Fig. 2a). Nevertheless, this loss of ancestral genes was accompanied by the acquisition of new ones by different mechanisms, including horizontal gene transfer (HGT). In the case of Vampirococcus, we detected, by phylogenetic analysis of all individual genes that had homologs

180 in other organisms, the acquisition of 126 genes by HGT from various donors (Extended Data Fig. 5).

The set of genes that could be annotated provided interesting clues about the biology and lifestyle of Vampirococcus. The most striking feature was its oversimplified energy and carbon metabolism map (Fig. 3). ATP generation in this CPR species appeared to depend entirely on

185 substrate-level phosphorylation carried out by the phosphoenolpyruvate kinase (EC 2.7.1.40). In fact, Vampirococcus only possesses incomplete glycolysis, which starts with 3-phosphoglycerate as first substrate. This molecule is the major product of the enzyme RuBisCO and, therefore, most likely highly available to Vampirococcus from its photosynthetic host. Comparison with nearly complete MAG sequences available for other Absconditabacteria/SR1 showed that 190 Vampirococcus has the most specialized carbon metabolism, with 3-phosphoglycerate as the 
only exploitable substrate, whereas the other species have a few additional enzymes that allow them to use other substrates (such as ribulose-1,5P and acetyl-CoA) as energy and reducing power (NADH) sources (Extended Data Fig. 6). This metabolic diversification probably reflects their adaptation to other types of hosts where these substrates are abundant. Vampirococcus also 195 lacks all the enzymes involved in some Absconditabacteria/SR1 in the 3-phosphoglyceratesynthesizing AMP salvage pathway ${ }^{19}$, including the characteristic archaeal-like type II/III RuBisCO ${ }^{20}$.

The genomes of Absconditabacteria/SR1 and Vampirococcus encode several electron carrier proteins (e.g., ferredoxin, cytochrome $b_{5}$, several Fe-S cluster proteins) and a membrane $\mathrm{F}_{1} \mathrm{Fo}_{\mathrm{O}}$ 200 type ATP synthase. However, they apparently lack any standard electron transport chain and, therefore, they seem to be non-respiring ${ }^{18,19,21}$. The electron carrier proteins may be related with the oxidative stress response and/or the reoxidation of reduced ferredoxin or $\mathrm{NADH}^{19}$. In the absence of any obvious mechanism to generate proton motive force (PMF), the presence of the membrane ATP synthase is also intriguing. It has been speculated either that CPR bacteria might 205 tightly adhere to their hosts and scavenge protons from them or that the membrane ATP synthase might work in the opposite direction as an ATPase, consuming ATP generated by substrate level phosphorylation to extrude protons and drive antiporters ${ }^{9}$. However, in the case of Vampirococcus the direct transport of protons from the host is unlikely since, as observed in the TEM sections (Fig. 1h), it seems that there is no direct contact with the host cell membrane.

210 Parasite and host cell membranes are separated by a relatively large space of $\sim 100 \mathrm{~nm}$, which would be largely conducive to proton diffusion and inefficient transfer between cells. Although the proton/cation antiporters (e.g., for $\mathrm{Na}^{+}, \mathrm{K}^{+}$or $\mathrm{Ca}^{+}$) encoded by Vampirococcus and the other Absconditabacteria/SR1 may serve to produce some PMF, it is improbable that this mechanism represents a major energy transducing system as cells would accumulate cations and disrupt their

215 ionic balance; these antiporters are most likely involved in cation homeostasis. This prompted us to investigate other ways that these cells might use to generate PMF usable by their ATP synthase. We found a protein (Vamp_33_45) with an atypical tripartite domain structure. The Nterminal region, containing 8 transmembrane helices, showed similarity with several flavocytochromes capable of moving electrons and/or protons across the plasma membrane

220 (e.g., ${ }^{22}$ ). The central part of the protein was a rubredoxin-like nonheme iron-binding domain likely able to transport electrons. Finally, the C-terminal region, containing an NAD-binding 
motif, was similar to ferredoxin reductases involved in electron transfer ${ }^{23}$. This unusual Vampirococcus 3-domain protein is well conserved in the other Absconditabacteria/SR1 genomes sequenced so far, suggesting it plays an important function in this CPR phylum. Its

225 architecture suggests that it can transport electrons and/or protons across the membrane using ferredoxin as electron donor and makes it a strong candidate to participate in a putative new PMF-generating system. Alternatively, this protein could play a similar role to that of some oxidoreductases in the strict anaerobic archaeon Thermococcus onnurineus, including a thioredoxin reductase, which couple reactive oxygen species detoxification with NAD(P)+ 230 regeneration from $\mathrm{NAD}(\mathrm{P}) \mathrm{H}$ to maintain the intracellular redox balance and enhance $\mathrm{O}_{2-}$ mediated growth despite the absence of heme-based or cytochrome-type proteins ${ }^{24}$.

Despite our Vampirococcus genome sequence appears to be complete, genes encoding enzymes involved in the biosynthesis of essential cell building blocks such as amino acids, nucleotides and nucleosides, cofactors, vitamins, and lipids are almost completely absent (Fig.

235 2b). Therefore, the classical bacterial metabolic pathways for their synthesis ${ }^{25}$ do not operate in Vampirococcus. Such extremely simplified metabolic potential, comparable to that of intracellular parasitic bacteria such as Mycoplasma ${ }^{26}$, implies that Vampirococcus must acquire these molecules from an external source and supports the predatory nature of the interaction with its photosynthetic host. An intriguing aspect of this interaction concerns the transfer of substrates

240 from the host to Vampirococcus, especially considering that, despite examination of several serial ultrathin sections, the cell membranes of these two partners do not appear to be in direct contact (Fig. 1h). Vampirococcus encodes several virulence factors, including divergent forms of hemolysin and hemolysin translocator (Vamp_11_169 and Vamp_9_166, respectively), a phage holin (Vamp_5_129), and a membrane-bound lytic murein transglycosylase (Vamp_144_2).

245 These proteins are likely involved in the host cell wall and membrane disruption leading to cell content release. Hemolysin has also been found in Saccharibacteria (formerly candidate phylum TM7), the only CPR phylum for which an epibiotic parasitic lifestyle has been demonstrated so $\operatorname{far}^{12,13}$. Recent coupled lipidomic-metagenomic analyses have shown that CPR bacteria that lack complete lipid biosynthesis are able to recycle membrane lipid from other bacteria ${ }^{27}$. In

250 Vampirococcus, also devoid of phospholipid synthesis, a phospholipase gene (Vamp_34_196) predicted to be secreted and that has homologs involved in host phospholipid degradation in several parasitic bacteria ${ }^{28}$, may not only help disrupting the host membrane but also to generate 
a local source of host phospholipids that it can use to build its own cell membrane. Two Vampirococcus peptidoglycan hydrolases (Vamp_68-56_103 and Vamp_145_30) and one 255 murein DD-endopeptidase (Vamp_311_38), also predicted to be secreted, most probably contribute to degrade the host cell wall.

Vampirococcus also possesses a number of genes encoding transporters, most of them involved in the transport of inorganic molecules (Fig. 3). One notable exception is the competence-related integral membrane protein ComEC (Vamp_67_106) ${ }^{29}$ which, together with

260 ComEA (Vamp_21_186) and type IV pili (see below), probably plays a role in the uptake of host DNA that, once transported into the epibiont, can be degraded by various restriction endonucleases (five genes encoding them are present) and recycled to provide the nucleotides necessary for growth (Extended Data Fig. 7). These proteins are widespread in other CPR bacteria where they may have a similar function ${ }^{30}$. Vampirococcus also encodes an ABC-type 265 oligopeptide transporter (Vamp_40_40) and a DctA-like C4-dicarboxylate transporter (Vamp_41_97), known to catalyze proton-coupled symport of several Krebs cycle dicarboxylates (succinate, fumarate, malate, and oxaloacetate) ${ }^{31}$. The first, coupled with the numerous peptidases present in Vampirococcus, most likely is a source of amino acids. By contrast, the role of DctA is unclear since Vampirococcus does not have a Krebs cycle.

270 In sharp contrast with its simplified central metabolism, Vampirococcus possesses genes related to the construction of an elaborate cell surface, which seems to be a common theme in many CPR bacteria ${ }^{9,11}$. They include genes involved in peptidoglycan synthesis, several glycosyltransferases, a Sec secretion system, and a rich repertoire of type IV pilus proteins. The retractable type IV pili are presumably involved in the tight attachment of Vampirococcus to its

275 host and in DNA uptake in cooperation with the ComEC protein. Other proteins probably play a role in the specific recognition and fixation to the host, including several very large proteins. In fact, the Vampirococcus membrane proteome is enriched in giant proteins. The ten longest predicted proteins (between 1392 and 4163 aa, see Extended Data Fig. 8) are inferred to have a membrane localization and are probably responsible of the conspicuous fibrous aspect of its cell 280 surface (Fig. 1i). Most of these proteins possess domains known to be involved in the interaction with other molecules, including protein-protein (WD40, TRP, and PKD domains) and proteinlipid (saposin domain) interactions and cell adhesion (DUF11, integrin, and fibronectin domains). Two other large membrane proteins (Vamp_6_203, 2368 aa, and Vamp_19_245, 1895 
aa) may play a defensive role as they contain alpha-2-macroglobulin protease-inhibiting domains

285 that can protect against proteases released by the host. Several other smaller proteins complete the membrane proteome of Vampirococcus, some of them also likely involved in recognition and attachment to the host thanks to a variety of protein domains, such as VWA (Vamp_41_85) and flotillin (Vamp_11_100).

\section{New CRISPR-Cas systems and other defense mechanisms in Vampirococcus}

Although most CPR phyla are devoid of CRISPR-Cas ${ }^{32}$, some have been found to contain new systems with original effector enzymes such as $\mathrm{CasY}^{33}$. In contrast with most available Absconditabacteria genomes, Vampirococcus possesses two CRISPR-Cas loci (Fig. 4a and Extended Data Fig. 9). The first is a class II type V system that contains genes coding for Cas1,

295 Cas2, Cas4, and Cpf1 proteins associated to 34 spacer sequences of 26-32 bp. Proteins similar to those of this system are encoded not only in genomes of close relatives of the Absconditabacteria (Gracilibacteria and Peregrinibacteria) but in many other CPR phyla. These sequences form monophyletic groups in phylogenetic analyses (e.g., Cas1, see Fig. 4b), which suggests that this type V system is probably ancestral in these CPR. The second system found in Vampirococcus

300 belongs to the class I type III and contains genes coding for Cas1, Cas2, Csm3, and Cas10/Csm1 proteins associated to a cluster of 20 longer (35-46 bp) spacers. In contrast with the previous CPR-like system, the proteins of this second system did not show strong similarity with any CPR homologue but with sequences from other bacterial phyla, suggesting that they have been acquired by HGT. Phylogenetic analysis confirmed this and supported that Vampirococcus 305 gained this CRISPR-Cas system from different distant bacterial donors (Extended Data Fig. 10). Interestingly, these two CRISPR-Cas systems encode a number of proteins that may represent new effectors. A clear candidate is the large protein Vamp_48_93 (1158 aa), located between Cpf1 and Cas1 in the type V system (Fig. 4a), which contains a DNA polymerase III PolC motif. Very similar sequences can be found in a few other CPR (some Roizmanbacteria, 310 Gracilibacteria, and Portnoybacteria) and in some unrelated bacteria (Extended Data Fig. 11). As in Vampirococcus, the gene coding for this protein is contiguous to genes encoding different Cas proteins in several of these bacteria, including Roizmanbacteria, Omnitrophica, and the deltaproteobacterium Smithella sp. (Extended Data Fig. 11). This gene association, as well as the very distant similarity between this protein and Cpfl CRISPR-associated proteins of bacterial 
315 type V systems, supports that it is a new effector in type V CRISPR-Cas systems. Additional putative new CRISPR-associated proteins likely exist also in the Vampirococcus type III system (Fig. 4a). Three proteins encoded by contiguous genes (Vamp_21_116, Vamp_21_127, and Vamp_21_128) exhibit very distant similarity with type III-A CRISPR-associated RAMP (Repeat Associated Mysterious Proteins) Csm4, Csm5, and Csm6 sequences, respectively, and 320 most probably represent new RAMP subfamilies. To date, Absconditabacteria ${ }^{34}$ and Saccharibacteria $^{35}$ are the only CPR phyla for which phages have been identified. Because of its proximity to Absconditabacteria, Vampirococcus is probably infected by similar phages, so that the function of its CRISPR-Cas systems may be related to the protection against these genetic parasites. Nevertheless, we did not find any similarity between the Vampirococcus spacers and

325 known phage sequences, suggesting that it is infected by unknown phages. Alternatively, considering that Vampirococcus -as most likely many other CPR bacteria- seems to obtain nucleotides required for growth by uptaking host DNA, an appealing possibility is that the CRISPR-Cas systems participate in the degradation of the imported host DNA

Although CPR bacteria have been hypothesized to be largely depleted of classical defense 330 mechanisms $^{36}$, we found that Vampirococcus, in addition to the two CRISP-Cas loci, is endowed with various other protection mechanisms. These include an AbiEii-AbiEi Type IV toxinantitoxin system, also present in other CPR bacteria, which may offer additional protection against phage infection ${ }^{37}$ and several restriction-modification systems, with three type I, one type II and one type III restriction enzymes and eight DNA methylases. In addition to a defensive 335 role, these enzymes may also participate in the degradation of the host DNA. As in its sistergroups Absconditabacteria and Gracilibacteria ${ }^{5,18,19,38,39}$, Vampirococcus has repurposed the UGA stop codon to code for glycine. The primary function of this recoding remains unknown but it has been speculated that it creates a genetic incompatibility, whereby these bacteria would be "evolutionarily isolated" from their environmental neighbors, preventing their potential

340 competitors from acquiring their genomic innovations by $\mathrm{HGT}^{18}$. However, the opposite might be argued as well, since the UGA codon reassignment can protect Vampirococcus from foreign DNA expression upon uptake by leading to aberrant protein synthesis via read-through of the UGA stop with Gly insertion. This can be important for these CPR bacteria because they are not only impacted by phages ${ }^{34}$ but they most likely depend on host DNA import and degradation to 345 fulfill their nucleotide requirements. In that sense, it is interesting to note that the Vampirococcus 
ComEA protein likely involved in DNA transport ${ }^{40}$ is encoded within the class I type III CRISPR-Cas system (Fig. 4a).

\section{Concluding remarks}

350 Our phylogenomic analyses show that Vampirococcus lugosii, the first genomically characterized example of the rare "predatory" bacteria described several decades ago ${ }^{14}$, is closely related to members of the phylum Absconditabacteria. V. lugosii thus becomes the first species characterized for a large subgroup of CPR bacteria that also includes Gracilibacteria and Peregrinibacteria (Fig. 2a). Vampirococcus is a parasite that attaches to the surface of

355 anoxygenic photosynthetic gammaproteobacteria and kills its host by consumption of its cytoplasmic content. In agreement with this predatory nature, the Vampirococcus genome sequence codes for an extremely simplified metabolism, implying that it depends on its host to obtain all cell constituents (amino acids, lipids, nucleotides, nucleosides, etc.). By contrast, it codes for a complex cell surface, including peptidoglycan, many transporters, pili, and several

360 giant proteins likely involved in the recognition and attachment to the host. The sticky nature of the Vampirococcus cell surface seems crucial for an original transmission mode. As Vampirococcus cells divide, they form stalks of piling cells, therefore increasing the probability of apical cells to encounter and attach to another host cell. Parasite filaments can connect two host cells but also release apical cells that, upon attachment to the host, start dividing again.

365 Despite intensive observations of TEM thin sections, communication bridges between parasite and host, and between piling parasite cells, were never observed. This implies a unique feeding mode whereby the parasite partially degrades the host cell membrane, exploiting its membrane hydrolysis products and cytoplasmic content. In principle, this feeding mode would be only available for the parasite in immediate vicinity with the host cell. This would entail that, upon 370 cell division, stalk parasite cells would be inactive until they stick to another host cell. Alternatively, although more speculative, stalk parasite cells might depend on the same original host and feed from neighboring parasitic cells by some sort of facilitated diffusion through the fibrous cell envelope in a peculiar type of kin-feeding mode. Despite a clear trend towards metabolic simplification, Vampirococcus, as well as other related CPR bacteria, presents a high

375 degree of evolutionary innovation. It includes many genes that do not show any similarity to 
those in sequence databases and that lack known conserved domains. Nevertheless, in some cases the genomic context allows to propose possible functions for some of these genes, as in the case of new effector proteins associated to the CRISPR-Cas loci. Other interesting innovations have evolved through new unique combinations of protein domains, as in the case of the 380 tripartite domain protein putatively involved in the generation of a proton gradient across membrane trough an unconventional respiratory chain.

So far, only members of the CPR Saccharibacteria have been maintained in stable cultures, allowing the characterization of their parasitic relationship with various bacterial hosts ${ }^{12,13,41}$. Using a "reverse genomics" approach with specific antibodies and cell sorting with flow

385 cytometry, cultures of human oral Absconditabacteria/SR1 species mixed with other bacteria have been recently established but their poor growth rate did not enable the detailed characterization of their biology ${ }^{13}$. Although we did not achieve to get stable Vampirococcus cultures, the recurrent examination of living material with optical microscopy combined with electron microscopy observations and genome sequence analysis clearly demonstrate its

390 predatory nature and allowed us to infer several mechanisms for host exploitation. Similarities in gene content with relatives in the Absconditabacteria/SR1 group suggest that this whole clade is composed of predatory or parasitic species. They most likely infect a wide spectrum of bacterial hosts, both photosynthetic and non-photosynthetic, as deduced from their metabolic specializations and their occurrence in very different habitats, such as deep aquifers, lakes, and

395 the human body ${ }^{13,18,19}$. The same has been proved for the Saccharibacteria ${ }^{12,13}$, adding to the growing evidence for parasitism as the general lifestyle of CPR bacteria. The vast diversity and habitat distribution of these bacteria suggest that they may play an ecological role analogous to that of viruses by controlling the population size of their host bacteria.

\section{Taxonomic Appendix}

The genome sequence of a species labelled as "Candidatus Vampirococcus archaeovorus" already existed in GenBank (CP019384.1) and belonged to a parasite of methanogenic archaea ascribed to the candidate division OP3, now known as Omnitrophica ${ }^{42}$. However, the organism that we have studied in this work fits much better the original description of the genus 405 Vampirococcus as an epibiotic parasite of photosynthetic anoxygenic bacteria with flat stacked 
cells ${ }^{14}$. Therefore, because of historical priority reasons, the genus name Vampirococcus, from "vampire" (Serbian: vampir, blood-sucker) and "coccus" (Greek: coccus, grain or berry), must be retained for this organism and a different genus name needs to be proposed for the homonymous parasite of archaea affiliated to the Omnitrophica.

\section{Description of 'Candidatus Vampirococcus lugosii'}

Lugosii after Bela Lugosi (1882-1956), who played the role of the vampire in the iconic 1931's film "Dracula". Epibiotic bacterium that preys on anoxygenic photosynthetic gammaproteobacterial species of the genus Halochromatium. Non-flagellated, small flat rounded cells (500-600 nm diameter and 200-250 nm height) that form piles of up to 10 cells attached to

415 the surface of the host. Gram-positive cell wall structure. Complete genome sequence, GenBank/EMBL/DDBJ accession number XXXX.

\section{Methods}

Sample collection and processing. Microbial mats were sampled in December 2013 in the

420 Salada de Chiprana (NE Spain) permanent athalassic hypersaline lake (55.9 g total dissolved salt $1^{-1}, \mathrm{pH} 8.23$ ) at a depth of $\sim 50 \mathrm{~cm}$. Fragments of these microbial mats were maintained under artificial illumination in 201 plastic containers filled with filtered lake water $(<0.2 \mu \mathrm{m})$. We daily monitored the growth of planktonic bacteria in these containers by optical microscopy. After $\sim 3$ weeks, we observed a bloom of Chromatium-like anoxygenic photosynthetic bacteria and 425 noticed that many cells had smaller epibiotic cells attached to them. We collected individual consortia of host-epibiont cells using an Eppendorf PatchMan NP2 micromanipulator equipped with $6 \mu \mathrm{m}$-diameter microcapillaries (Eppendorf) mounted on a Leica D1113000 B inverted microscope. Consortia were rinsed twice with sterile $10 \mathrm{mM}$ Tris $\mathrm{pH} 8.0$ buffer and finally deposited in a volume of $0.5 \mu \mathrm{l}$ of this buffer and stored frozen at $-20^{\circ} \mathrm{C}$ until further processing.

430 Microscopy. Light microscopy observation of living material was carried out on a Zeiss Axioplan microscope equipped with a Nikon Coolpix B500 color camera. For Scanning Electron Microscopy (SEM), liquid samples enriched in Vampirococcus and its host were deposited on top of $0.1 \mu \mathrm{m}$ pore-diameter filters (Whatman) under a mild vacuum aspiration regime and briefly rinsed with $0.1-\mu \mathrm{m}$ filtered and autoclaved MilliQ water under the same vacuum regime. 
435 Filters were let dry and sputtered with gold. SEM observations were carried out using a Field Emission Electron Microscope Philips XL30 S-FEG. Secondary electron (SE2) images were acquired using an In Lens detector at an accelerating voltage of $2.0 \mathrm{kV}$ and a working distance of 7.5 mm. For Transmission Electron Microscopy (TEM), $1.5 \mathrm{ml}$ of the sample were centrifuged for 2 minutes at 10,000 rpm. Cells were fixed with $2 \%$ glutaraldehyde in $0.1 \mathrm{M}$ cacodylate at $\mathrm{pH}$

4407.4 and postfixed in $2 \%$ osmium tetroxide for 30 minutes. Cells were then dehydrated in a gradual series of ethanol baths $(50 \%, 70 \%$ and 100\%) and dried cells were embedded in epoxy resin. The resin block was cut into $90 \mathrm{~nm}$ thick sheets using an Ultracut UCT ultramicrotome. The sheets were placed on copper grids and observed in a JEM1010 (Jeol) microscope with an acceleration voltage of $100 \mathrm{KeV}$.

445 DNA extraction, 16S rRNA gene PCR amplification, and whole genome amplification. DNA was purified from individual (or few, ca. 10 cells) cell consortia with the PicoPure DNA extraction kit (Applied Biosystems). 16S rRNA genes were PCR-amplified using the primers B27F (AGAGTTTGATCCTGGCTCAG) and 1492R (GGTTACCTTGTTACGACTT). PCR reactions were performed for 30 cycles (denaturation at $94^{\circ} \mathrm{C}$ for $15 \mathrm{~s}$, annealing at $55^{\circ} \mathrm{C}$ for 30

$450 \mathrm{~s}$, extension at $72^{\circ} \mathrm{C}$ for $2 \mathrm{~min}$ ) preceded by 2 min denaturation at $94^{\circ} \mathrm{C}$, and followed by $7 \mathrm{~min}$ extension at $72^{\circ} \mathrm{C}$. $16 \mathrm{~S}$ rRNA gene clone libraries were constructed with the Topo TA cloning system (Invitrogen) following the instructions provided by the manufacturer. After plating, positive transformants were screened by PCR amplification using M13R and T7 flanking vector primers. 16S rRNA amplicons were Sanger-sequenced using the 1492R primer by Genewiz

455 (Essex, UK). Whole genome amplification (WGA) was carried out on PicoPure-extracted DNA using Multiple Displacement Amplification ${ }^{43}$ (MDA) with the REPLI-g WGA kit (Qiagen) and Multiple Annealing and Looping-Based Amplification Cycles $^{44}$ (MALBAC) with the MALBAC Single Cell WGA kit (Yikon Genomics).

Genome sequencing, assembly and annotation. Sequencing of WGA products from three 460 independent consortia with identical 16S rRNA gene sequences (V7 and V8, amplified by MDA, and V12, amplified by MALBAC) was done using Illumina HiSeq2500 v4 (2x125 bp paired-end reads) by Eurofins Genomics (Ebersberg, Germany). Each dataset was separately assembled into contigs with SPAdes v3.6. $0^{45}$ using default parameters. MDA contigs longer than $2.5 \mathrm{~kb}$ were clustered using the ESOM procedure (ESOM Tools v. $1.14^{46}$ ) along with 3 reference genomes 465 chosen for their close phylogenetic relation to Vampirococcus and its host: Candidate division 
SR1 bacterium RAAC1_SR1_1 (CP006913), Allochromatium vinosum DSM 180 (NC_013851), Thioflavicoccus mobilis 8321 (NC_019940), along with an external reference: Escherichia coli BL21-Gold(DE3)pLysS AG (NC_012947). The clustering was done on nucleotide tetramer frequency distributions computed with the tetramer_freqs_esom.pl script ${ }^{6}$ using a window size of $4705 \mathrm{~kb}$. ESOM training parameters where set to k-batch training method, $140 \times 250$ mesh size, radius start 50, 20 training epochs. All other parameters were set to their defaults. The visualization of the ESOM maps was done with a U-Matrix background and inverse gray-scale gradient coloring (Extended Data Fig. 12). Further improvement was achieved by crossmatching the MDA ESOM-processed sequence set with contigs from the MALBAC set, which

475 increased the Vampirococcus total sequence size by $\sim 10 \%$. The completeness and contamination of the bins were assessed using CheckM $^{47}$.

CDS prediction was performed on the assembled contigs using Prodigal version 2.6.2 (single mode, translation table 25 (Candidate Division SR1 and Gracilibacteria) ${ }^{48}$. For more accurate functional annotation, we submitted the amino acid sequence of predicted genes to the blastp 480 command of DIAMOND version 0.7 .9 (maximum e-value of $10-5)^{49}$ to search three databases: the non-redundant protein RefSeq (release 68; Nov 3, 2014), $\mathrm{COG}^{50}$, and SEED (release of September 14, 2011) ${ }^{51}$. Enzymes involved in metabolic pathways were also searched against the KEGG database ${ }^{25}$. Conserved protein motifs were searched using SMART $^{52}$. Prediction of transmembrane helices was done with the TMHMM Server v. 2.0

485 (http://www.cbs.dtu.dk/services/TMHMM/). CRISPR-Cas loci were identified using CRISPRCasFinder ${ }^{53}$ and CRISPRminer ${ }^{54}$. Ancestral gene content and gene loss in the group Absconditabacteria + Gracilibacteria + Peregrinibacteria were inferred by first carrying out orthologue clustering with OrthoFinder v1.1.20 $0^{55}$ with default parameters, and then applying the Dollo parsimony method implemented in the Count software ${ }^{56}$.

490 Phylogenetic Analyses. 16S rRNA and protein sequences were aligned using MAFFT L-INS-i ${ }^{57}$ and poorly aligned regions were removed with trimAl -automated $1^{58}$. Maximum Likelihood (ML) phylogenetic trees were reconstructed using IQ-tree v. 1.5.54959 with the GTR+G+I model for $16 \mathrm{~S}$ rRNA gene alignments and the $\mathrm{LG}+\mathrm{C} 20+\mathrm{G}$ model for individual protein alignments. Multi-protein datasets were constructed by concatenation of individual protein trimmed 495 alignments using SequenceMatrix ${ }^{60}$. ML trees of multi-protein datasets were inferred using IQtree and the $\mathrm{LG}+\mathrm{PMSF}(\mathrm{C} 60)+\mathrm{F}+\mathrm{G} 4$ model with a guide tree inferred with the $\mathrm{LG}+\mathrm{C} 60+\mathrm{F}+\mathrm{G} 4$ 
model. In all cases, branch support was estimated with the bootstrap method (100 replicates) implemented in IQ-tree.

\section{References}

1. López-García, P. \& Moreira, D. Tracking microbial biodiversity through molecular and genomic ecology. Res. Microbiol. 159, 67-73 (2008).

2. Hug, L.A. et al. A new view of the tree of life. Nat. Microbiol. 1, 16048 (2016).

3. Solden, L., Lloyd, K. \& Wrighton, K. The bright side of microbial dark matter: lessons learned from 505 the uncultivated majority. Curr. Opin. Microbiol. 31, 217-226 (2016).

4. Castelle, C.J. \& Banfield, J.F. Major new microbial groups expand diversity and alter our understanding of the Tree of Life. Cell 172, 1181-1197 (2018).

5. Rinke, C. et al. Insights into the phylogeny and coding potential of microbial dark matter. Nature 499, 431-437 (2013).

510 6. Dick, G.J. et al. Community-wide analysis of microbial genome sequence signatures. Genome Biol. 10, R85 (2009).

7. Zaremba-Niedzwiedzka, K. et al. Asgard archaea illuminate the origin of eukaryotic cellular complexity. Nature 541, 353-358 (2017).

$515 \quad$ Nature 523, 208-211 (2015).

9. Castelle, C.J. et al. Biosynthetic capacity, metabolic variety and unusual biology in the CPR and DPANN radiations. Nat. Rev. Microbiol. 16, 629-645 (2018).

10. Dombrowski, N., Lee, J.H., Williams, T.A., Offre, P. \& Spang, A. Genomic diversity, lifestyles and evolutionary origins of DPANN archaea. FEMS Microbiol. Lett. 366, fnz008 (2019).

520 11. Luef, B. et al. Diverse uncultivated ultra-small bacterial cells in groundwater. Nat. Commun. 6, 6372 (2015).

12. He, X. et al. Cultivation of a human-associated TM7 phylotype reveals a reduced genome and epibiotic parasitic lifestyle. Proc. Natl. Acad. Sci. U. S. A. 112, 244-249 (2015).

$525 \quad$ Nat. Biotechnol. 37, 1314-1321 (2019).

14. Guerrero, R. et al. Predatory prokaryotes: predation and primary consumption evolved in bacteria. Proc. Natl. Acad. Sci. U. S. A. 83, 2138-42. (1986).

15. De Wit, R. Lake La Salada de Chiprana (NE Spain), an example of an athalassic salt lake in a cultural landscape. in Lake Sciences and Climate Change (ed. Nageeb-Rashed, M.) 43-60 (Intech ed., London, 2016).

16. Creevey, C.J., Doerks, T., Fitzpatrick, D.A., Raes, J. \& Bork, P. Universally distributed single-copy genes indicate a constant rate of horizontal transfer. PLoS One 6, e22099 (2011).

17. Nelson, W.C. \& Stegen, J.C. The reduced genomes of Parcubacteria (OD1) contain signatures of a symbiotic lifestyle. Front. Microbiol. 6, 713 (2015).

535 18. Campbell, J.H. et al. UGA is an additional glycine codon in uncultured SR1 bacteria from the human microbiota. Proc. Natl. Acad. Sci. U. S. A. 110, 5540-5545 (2013).

19. Kantor, R.S. et al. Small genomes and sparse metabolisms of sediment-associated bacteria from four candidate phyla. MBio 4, 00708-00713 (2013).

$540 \quad$ metabolism. Science. 315, 1003-1006. (2007).

21. Dueholm, M.S. et al. Complete genome sequence of the bacterium Aalborg_AAW-1, representing a novel family within the Candidate Phylum SR1. Genome Announc. 3, e00624-15 (2015). 
22. Loschi, L. et al. Structural and biochemical identification of a novel bacterial oxidoreductase. J. Biol. Chem. 279, 50391-50400. (2004).

545 23. Arakaki, A.K., Ceccarelli, E.A. \& Carrillo, N. Plant-type ferredoxin-NADP+ reductases: a basal structural framework and a multiplicity of functions. Faseb J. 11, 133-140 (1997).

24. Lee, S.H., Youn, H., Kang, S.G. \& Lee, H.S. Oxygen-mediated growth enhancement of an obligate anaerobic archaeon Thermococcus onnurineus NA1. J. Microbiol. 57, 138-142 (2019).

\section{1611, 135-145 (2017).}

26. Fraser, C.M. et al. The minimal gene complement of Mycoplasma genitalium. Science. 270, 397403. (1995).

27. Probst, A.J. et al. Lipid analysis of $\mathrm{CO}(2)$-rich subsurface aquifers suggests an autotrophy-based deep biosphere with lysolipids enriched in CPR bacteria. ISME J. 14, 1547-1560 (2020).

555 28. Toledo, A. \& Benach, J.L. Hijacking and use of host lipids by intracellular pathogens. Microbiol. Spectr. 3, 0001-2014 (2015).

29. Hahn, J., Inamine, G., Kozlov, Y. \& Dubnau, D. Characterization of comE, a late competence operon of Bacillus subtilis required for the binding and uptake of transforming DNA. Mol. Microbiol. 10, 99-111. (1993).

560 30. Meheust, R., Burstein, D., Castelle, C.J. \& Banfield, J.F. The distinction of CPR bacteria from other bacteria based on protein family content. Nat. Commun. 10, 4173 (2019).

31. Groeneveld, M., Weme, R.G., Duurkens, R.H. \& Slotboom, D.J. Biochemical characterization of the C4-dicarboxylate transporter DctA from Bacillus subtilis. J. Bacteriol. 192, 2900-2907 (2010).

565 systems. Nat. Commun. 7, 10613 (2016).

33. Chen, L.X. et al. Candidate Phyla Radiation Roizmanbacteria from hot springs have novel and unexpectedly abundant CRISPR-Cas systems. Front. Microbiol. 10, 928 (2019).

34. Paez-Espino, D. et al. Uncovering Earth's virome. Nature 536, 425-430 (2016).

35. Dudek, N.K. et al. Novel microbial diversity and functional potential in the marine mammal oral microbiome. Curr. Biol. 27, 3752-3762 (2017).

36. Koonin, E.V., Makarova, K.S. \& Wolf, Y.I. Evolutionary genomics of defense systems in Archaea and Bacteria. Annu. Rev. Microbiol. 71, 233-261 (2017).

37. Dy, R.L., Przybilski, R., Semeijn, K., Salmond, G.P. \& Fineran, P.C. A widespread bacteriophage abortive infection system functions through a Type IV toxin-antitoxin mechanism. Nucleic Acids

575 Res. 42, 4590-4605 (2014).

38. Wrighton, K.C. et al. Fermentation, hydrogen, and sulfur metabolism in multiple uncultivated bacterial phyla. Science 337, 1661-1665 (2012).

39. Hanke, A. et al. Recoding of the stop codon UGA to glycine by a BD1-5/SN-2 bacterium and niche partitioning between Alpha- and Gammaproteobacteria in a tidal sediment microbial community

580 naturally selected in a laboratory chemostat. Front. Microbiol. 5, 231 (2014).

40. Seitz, P. et al. ComEA is essential for the transfer of external DNA into the periplasm in naturally transformable Vibrio cholerae cells. PLoS Genet. 10, e1004066 (2014).

41. Bor, B. et al. Insights obtained by culturing Saccharibacteria with their bacterial hosts. J. Dent. Res. 99, 685-694 (2020).

585 42. Kizina, J. et al. Insights into the biology of Candidate Division OP3 LiM populations. PhD thesis. Bremen, Germany, University of Bremen, 85-160 (2017).

43. Dean, F.B. et al. Comprehensive human genome amplification using multiple displacement amplification. Proc. Natl. Acad. Sci. U. S. A. 99, 5261-5266 (2002).

44. Zong, C., Lu, S., Chapman, A.R. \& Xie, X.S. Genome-wide detection of single-nucleotide and copy-

590 number variations of a single human cell. Science 338, 1622-1626 (2012). sequencing. J. Comput. Biol. 19, 455-477 (2012). 
46. Ultsch, A. \& Mörchen, F. ESOM-Maps: tools for clustering, visualization, and classification with Emergent SOM. (Tech. Rep. Dept. Math. Comput. Sci. Univ. Marburg, Ger., 2005).

47. Parks, D.H., Imelfort, M., Skennerton, C.T., Hugenholtz, P. \& Tyson, G.W. CheckM: assessing the quality of microbial genomes recovered from isolates, single cells, and metagenomes. Genome Res. 25, 1043-1055 (2015).

48. Hyatt, D. et al. Prodigal: prokaryotic gene recognition and translation initiation site identification. BMC Bioinformatics 11, 1471-2105 (2010).

600 49. Buchfink, B., Xie, C. \& Huson, D.H. Fast and sensitive protein alignment using DIAMOND. Nat. Methods 12, 59-60 (2015).

50. Galperin, M.Y., Makarova, K.S., Wolf, Y.I. \& Koonin, E.V. Expanded microbial genome coverage and improved protein family annotation in the COG database. Nucleic Acids Res. 43, D261-269 (2015).

605 51. Overbeek, R. et al. The SEED and the Rapid Annotation of microbial genomes using Subsystems Technology (RAST). Nucleic Acids Res. 42, D206-214 (2014).

52. Letunic, I. \& Bork, P. 20 years of the SMART protein domain annotation resource. Nucleic Acids Res. 46, D493-d496 (2018).

53. Couvin, D. et al. CRISPRCasFinder, an update of CRISRFinder, includes a portable version, enhanced performance and integrates search for Cas proteins. Nucleic Acids Res. 46, W246-w251 (2018).

54. Zhang, F. et al. CRISPRminer is a knowledge base for exploring CRISPR-Cas systems in microbe and phage interactions. Commun. Biol. 1, 180 (2018).

615 dramatically improves orthogroup inference accuracy. Genome Biol. 16, 157 (2015).

56. Csuros, M. Count: evolutionary analysis of phylogenetic profiles with parsimony and likelihood. Bioinformatics 26, 1910-1912 (2010).

57. Katoh, K. \& Standley, D.M. MAFFT multiple sequence alignment software version 7: improvements in performance and usability. Mol. Biol. Evol. 30, 772-780 (2013).

620 58. Capella-Gutierrez, S., Silla-Martinez, J.M. \& Gabaldon, T. trimAl: a tool for automated alignment trimming in large-scale phylogenetic analyses. Bioinformatics. 25, 1972-1973. (2009).

59. Nguyen, L.T., Schmidt, H.A., von Haeseler, A. \& Minh, B.Q. IQ-TREE: a fast and effective stochastic algorithm for estimating maximum-likelihood phylogenies. Mol. Biol. Evol. 32, 268-274 (2015).

625 60. Vaidya, G., Lohman, D.J. \& Meier, R. SequenceMatrix: concatenation software for the fast assembly of multi-gene datasets with character set and codon information. Cladistics 27, 171-180 (2011).

\section{Acknowledgements}

630 We thank M. Ragon and the UNICELL platform (http://www.deemteam.fr/en/unicell) for carrying out WGA experiments, and A. Saghaï for 16S rRNA gene amplification assays of Vampirococcus relatives. This work was supported by the European Research Council (ERC) Advanced Grants 'Protistworld' and 'Plast-Evol' (322669 and 787904, respectively). 
D.M., P.L.-G., A.I.L.-A. and M.I. carried out sampling and monitored Vampirococcus growth. A.I.L.-A. carried out electron microscopy observations. D.M. micromanipulated cells. P.L.-G. amplified 16S rRNA genes from isolated cells. Y.Z. assembled and annotated the Vampirococcus genome. D.M. analyzed gene sequences to infer metabolism and other cell 640 features. D.M. wrote the manuscript with help from the rest of co-authors. 


\section{Figure legends}

Fig. 1 | Sampling site and microscopy observation of Vampirococcus cells. a, General view of

650 the microbial mat covering the shore of the Salada de Chiprana lake. b, Closer view of a microbial mat section. c, Natural population of blooming sulfide-dependent anoxygenic photosynthetic bacteria in waters of microbial mat containers after several weeks of growth in the laboratory; note the conspicuous refringent intracellular sulfur inclusions. d-f, Closer microscopy view of anoxygenic photosynthetic bacteria infected by epibiotic Vampirococcus

655 cells and few-cell filaments (indicated by yellow arrows). g, Scanning electron microscopy image of a host cell infected by two stacking cells of Vampirococcus (yellow arrow). h, Transmission electron microscopy (TEM) image of a thin section of a host cell infected by Vampirococcus (yellow arrow). i, Closer TEM view of a thin section of Vampirococcus cells, notice the fibrous rugose cell surface and the large space separating contiguous cells. Scale bars:

$6605 \mathrm{~cm}(\mathrm{~b}), 5 \mu \mathrm{m}$ (c), $1 \mu \mathrm{m}(\mathrm{d}-\mathrm{h}), 0.5 \mu \mathrm{m}$ (i).

Fig. 2 | Phylogeny and global gene content of the Vampirococcus genome. a, Maximum likelihood phylogenetic tree of bacteria based on a concatenated dataset of 16 ribosomal proteins showing the position of Vampirococcus lugosii close to the Absconditabacteria (for the complete 665 tree, see Extended Data Fig. 4). Histograms on the right show the proportion of genes retained in each species from the ancestral pool inferred for the last common ancestor of Absconditabacteria, Gracilibacteria and Peregrinibacteria. b, Percentage of Vampirococcus genes belonging to the different COG categories. c, Genes shared by Vampirococcus and the three Absconditabacteria genomes shown in the phylogenetic tree. COG categories are: Energy

670 production and conversion [C]; Cell cycle control, cell division, chromosome partitioning [D]; Amino acid transport and metabolism [E]; Nucleotide transport and metabolism [F]; Carbohydrate transport and metabolism [G]; Coenzyme transport and metabolism [H]; Lipid transport and metabolism [I]; Translation, ribosomal structure and biogenesis [J]; Transcription [K]; Replication, recombination and repair [L]; Cell wall/membrane/envelope biogenesis [M];

675 Secretion, motility and chemotaxis $[\mathrm{N}]$; Posttranslational modification, protein turnover, chaperones [O]; Inorganic ion transport and metabolism [P]; General function prediction only 
[R]; Function unknown [S]; Intracellular trafficking, secretion, and vesicular transport [U]; Defense mechanisms [V]; Mobilome: prophages, transposons [X]; Secondary metabolites biosynthesis, transport and catabolism [Q].

680

Fig. 3 | Metabolic and cell features inferred from the genes encoded in the Vampirococcus genome. The diagram shows the host cell surface (bottom) with two stacking Vampirococcus cells attached to its surface (as in Fig. 1h).

685 Fig. 4 | CRISPR-Cas systems in Vampirococcus. a, Genes in the two systems encoded in the Vampirococcus lugosii genome. b, Maximum likelihood phylogenetic tree of the Cas1 protein encoded in the class II type V system, numbers at branches indicate bootstrap support. 
a

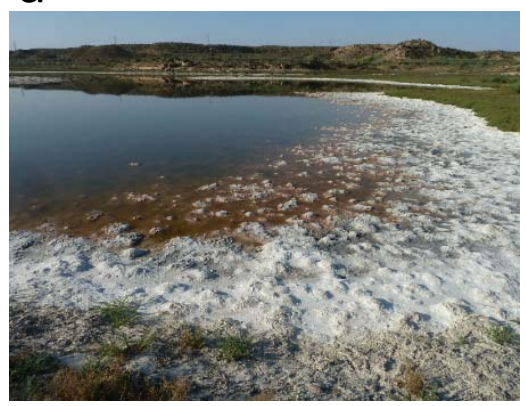

d
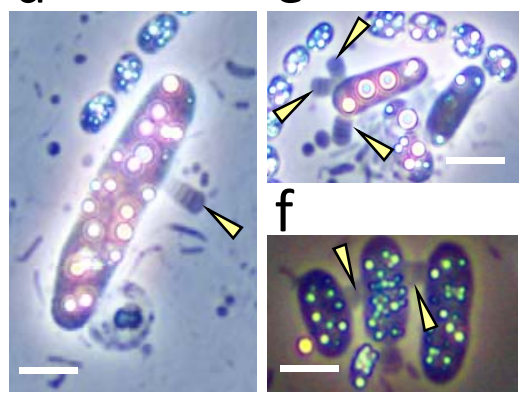

b

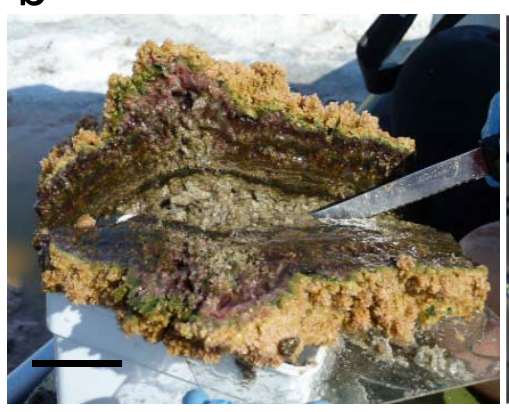

g

h

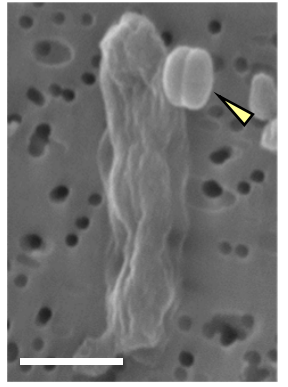

C

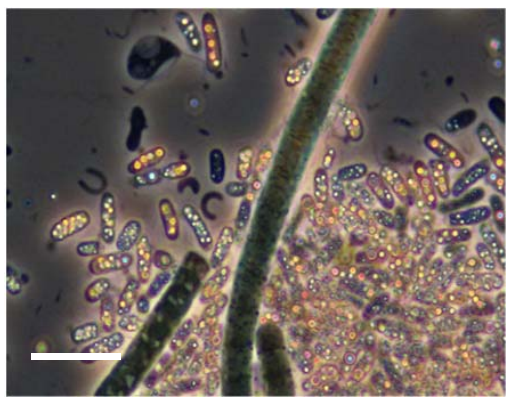

i
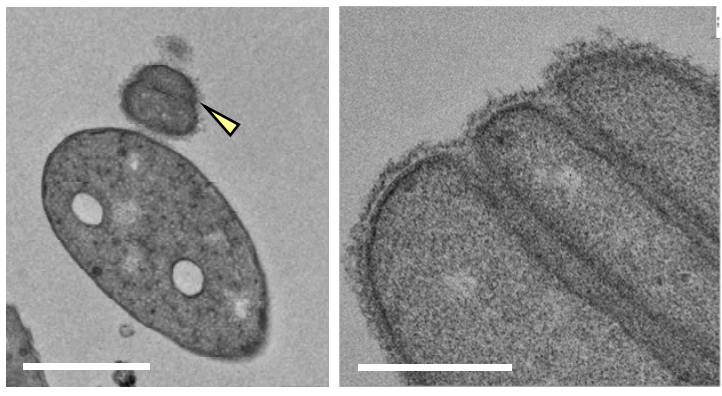
bioRxiv preprint doi: https://doi.org/10.1101/2020.11.10.374967; this version posted November 10, 2020. The copyright holder for this preprint (which was not certified by peer review) is the author/funder. All rights reserved. No reuse allowed without permission.

a

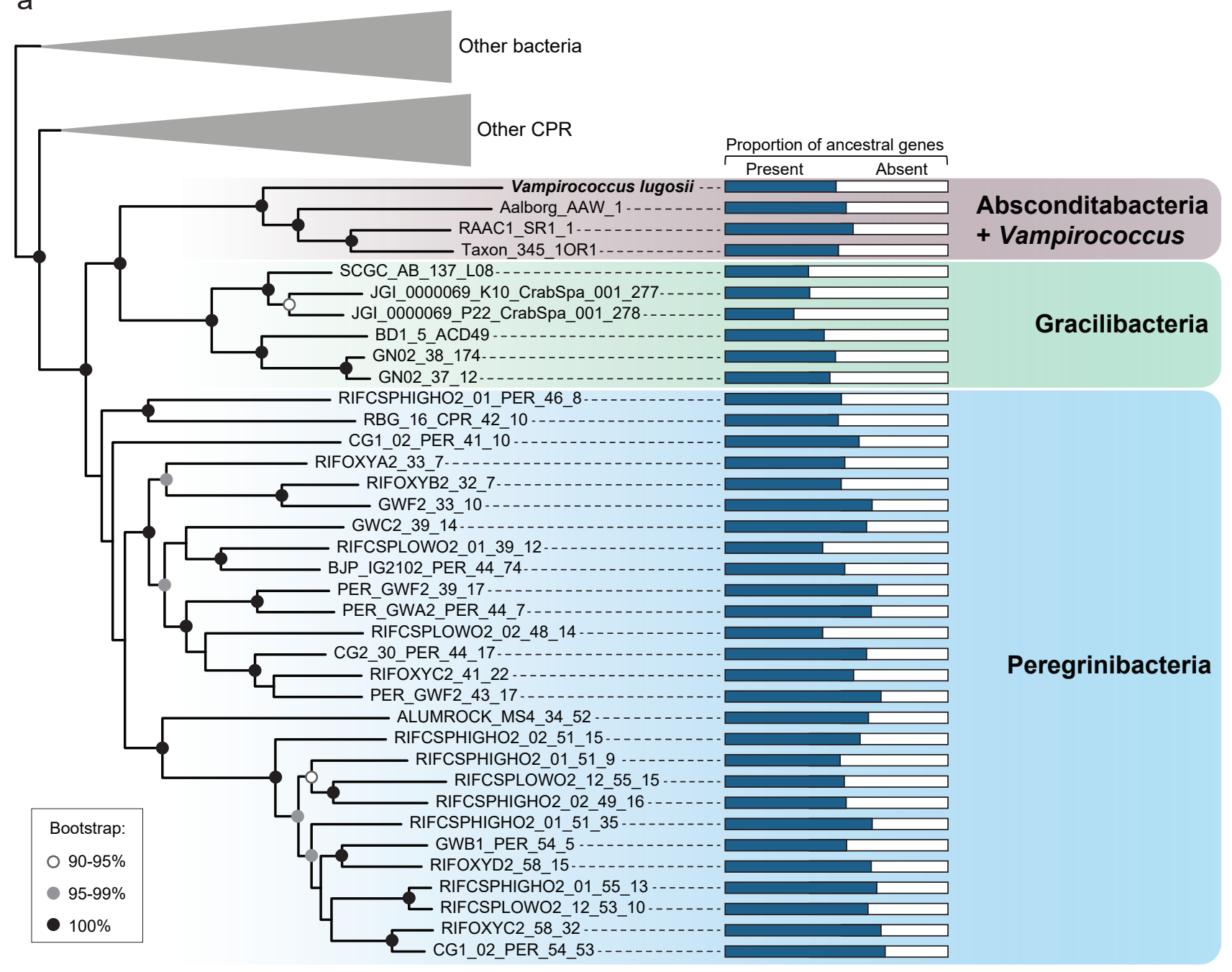

b

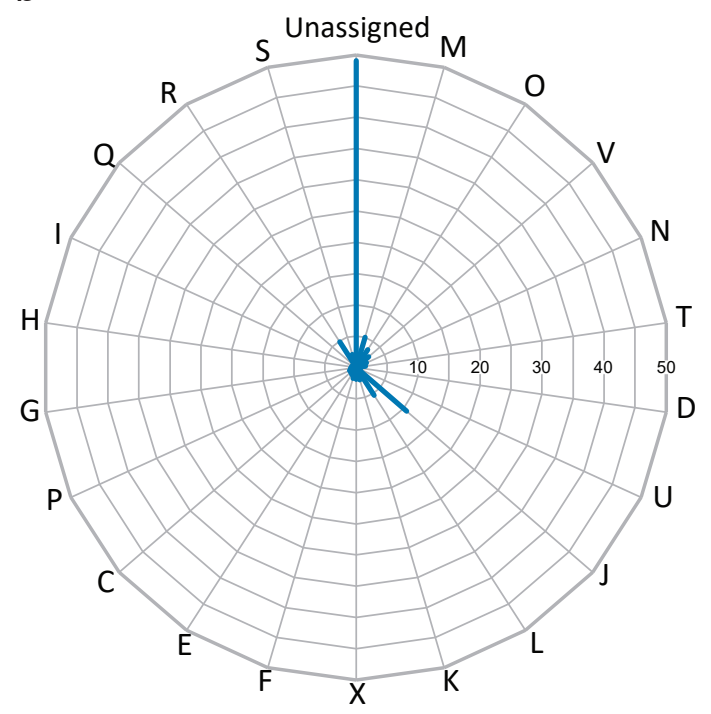

C

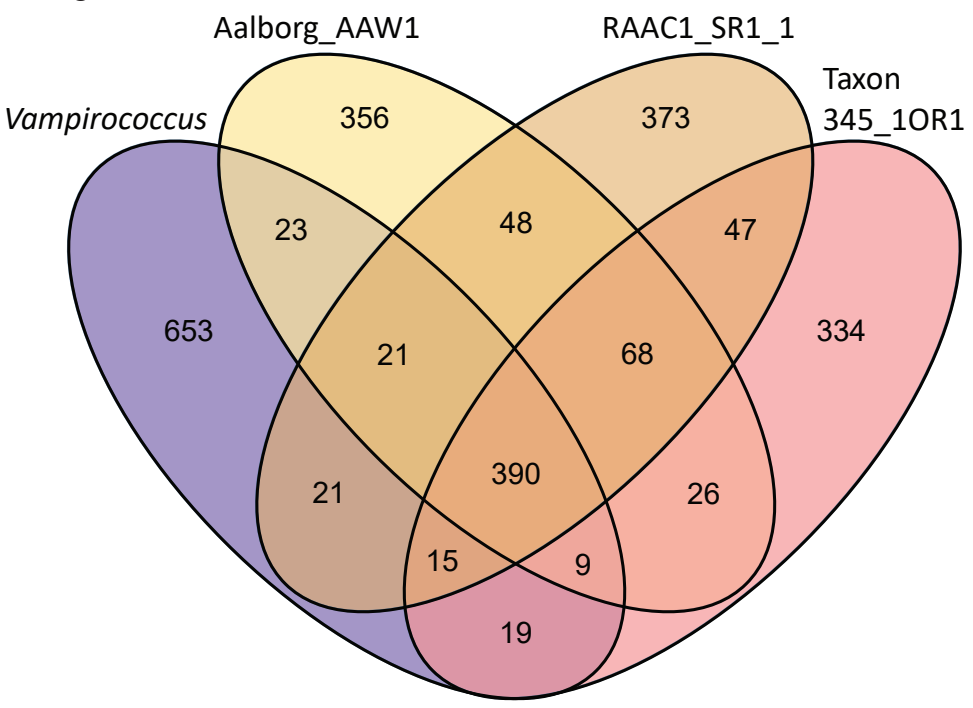




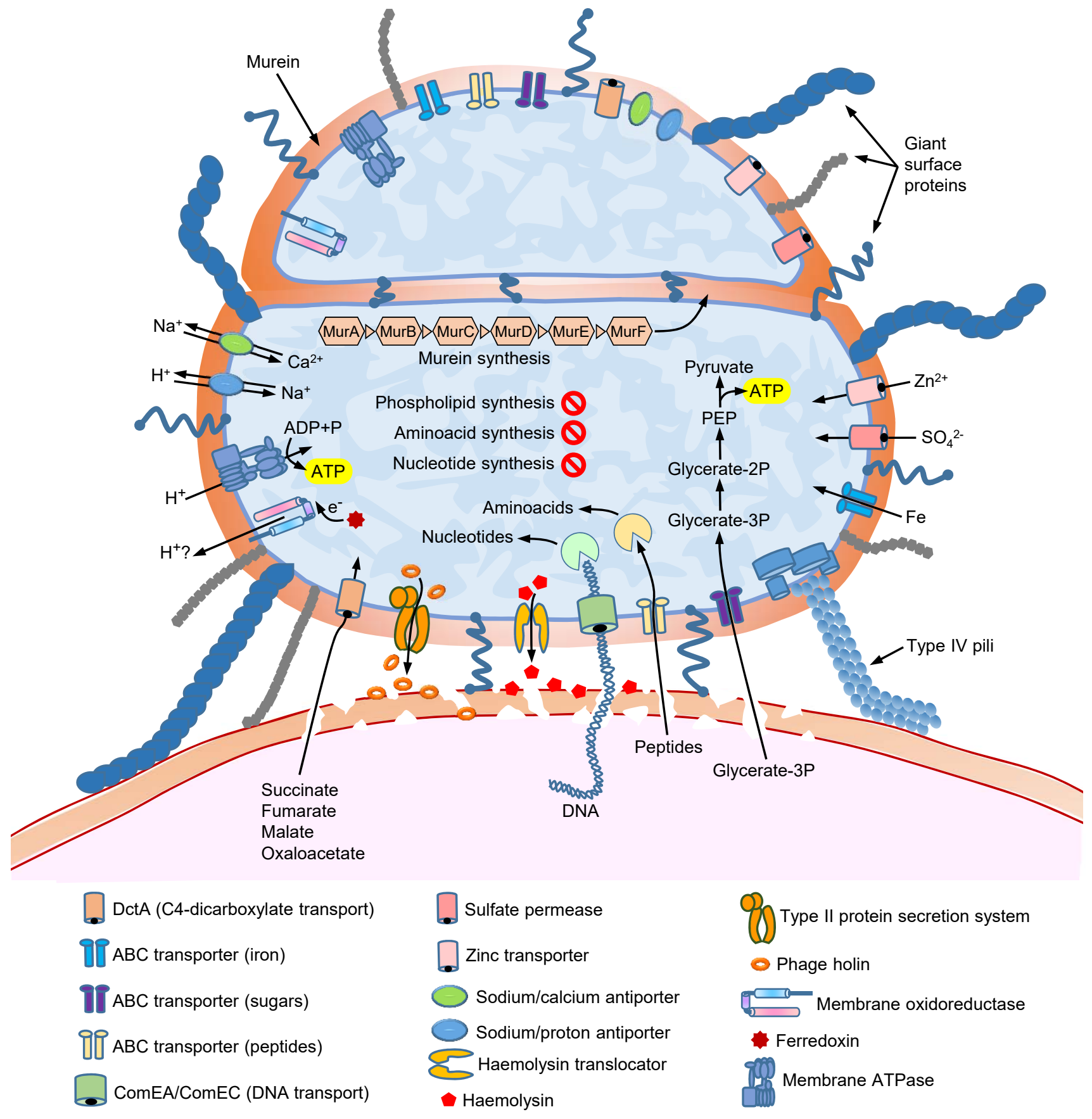


bioRxiv preprint doi: https://doi.org/10.1101/2020.11.10.374967; this version posted November 10, 2020. The copyright holder for this preprint a (which was not certified by peer review) is the author/funder. All rights reserved. No reuse allowed without permission.

\section{Class II type V}

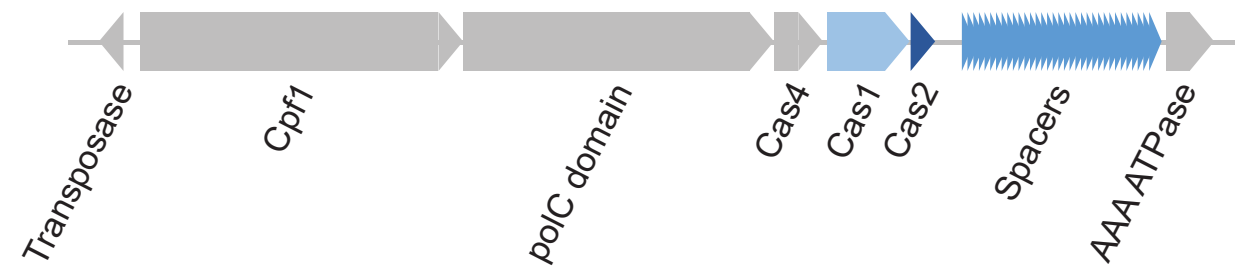

\section{Class I type III}

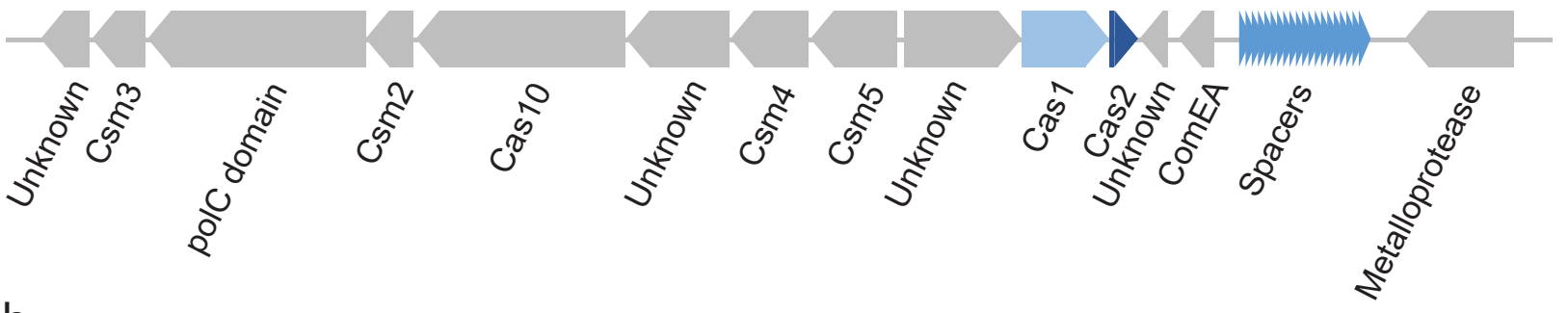

b

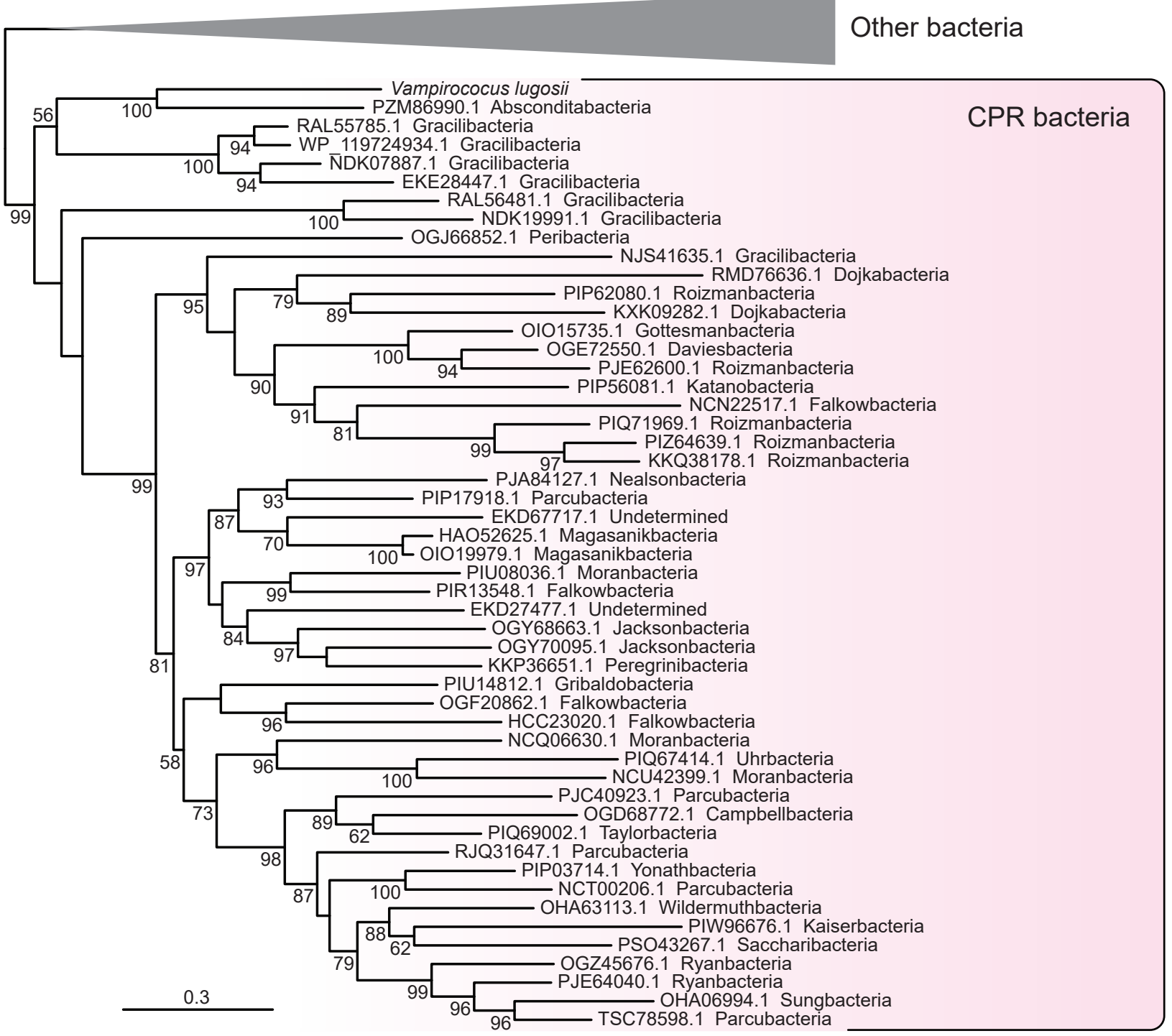

